\section{(6) OPEN ACCESS}

\title{
Surgical management of aortic root disease in Marfan syndrome and other congenital disorders associated with aortic root aneurysms
}

\author{
Tom Treasure, ${ }^{1}$ J J M Takkenberg, ${ }^{2}$ John Pepper ${ }^{3,4}$
}

\begin{abstract}
${ }^{1}$ Clinical Operational Research Unit, University College London, London, UK ${ }^{2}$ Department of CardioThoracic Surgery, Erasmus University Medical Center, Rotterdam, The Netherlands ${ }^{3} \mathrm{NIHR}$ Cardiovascular BRU, Royal Brompton Hospital, London, UK

${ }^{4}$ Institute of Cardiovascular Medicine and Science (ICMS), London, UK
\end{abstract}

\section{Correspondence to} Professor Tom Treasure, Clinical Operational Research Unit, University College London, 4 Taviton Street, London WC1H OBT, UK: tom.treasure@gmail.com

Received 22 January 2014 Revised 30 May 2014 Accepted 3 June 2014 Published Online First 1 July 2014
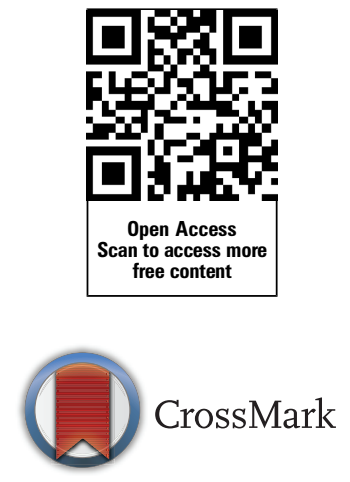

\footnotetext{
To cite: Treasure $\mathrm{T}$, Takkenberg JJM, Pepper J. Heart 2014;100:15711576.
}

\begin{abstract}
Elective root replacement in Marfan syndrome has improved life expectancy in affected patients. Three forms of surgery are now available: total root replacement (TRR) with a valved conduit, valve sparing root replacement (VSRR) and personalised external aortic root support (PEARS) with a macroporous mesh sleeve. TRR can be performed irrespective of aortic dimensions and a mechanical replacement valve is a secure and near certain means of correcting aortic valve regurgitation but has thromboembolic and bleeding risks. VSRR offers freedom from anticoagulation and attendant risks of bleeding but reoperation for aortic regurgitation runs at $1.3 \%$ per annum. A prospective multi-institutional study has found this to be an underestimate of the true rate of valve-related adverse events. PEARS conserves the aortic root anatomy and optimises the chance of maintaining valve function but average follow-up is under 5 years and so the long-term results are yet to be determined. Patients are on average in their 30 s and so the cumulative lifetime need for reoperation, and of any valve-related complications, are consequently substantial. With lowering surgical risk of prophylactic root replacement, the threshold for intervention has reduced progressively over 30 years to $4.5 \mathrm{~cm}$ and so an increasing number of patients who are not destined to have a dissection are now having root replacement. In evaluation of these three forms of surgery, the number needed to treat to prevent dissection and the balance of net benefit and harm in future patients must be considered.
\end{abstract}

Aortic dissection is by far the commonest cause of death in the natural history of Marfan syndrome and other genetically defined aortopathies (figure 1). When there is an aortic root aneurysm, prophylactic surgery on the aortic root intended to prevent aortic dissection is the most important life prolonging treatment for individuals with these conditions and will therefore be given the most attention within the limitations of this clinical review.

Among the total number of patients with root aneurysms, those with a diagnosis of Marfan syndrome dominate the younger age range but they are nevertheless a minority of all patients with ascending aortic aneurysm ${ }^{1}$ (figure 2). They are prone to acute dissection and prior to the introduction of prophylactic root replacement this was the cause of death in two-thirds of all patients and often at a young age..$^{2-4}$ Detection, diagnosis, monitoring and prophylactic surgery are believed to have dramatically reduced deaths from dissection and to have resulted in increased longevity in these patients.

\section{THE APPROACH TO PREVENTION OF ACUTE ASCENDING AORTIC DISSECTION IN MARFAN SYNDROME \\ The threshold for elective intervention and the NNtT}

The genetic aortopathies are of their nature usually clustered in known affected families. These families are increasingly well informed and highly motivated to have their relatives and children assessed and to attend for echo measurements. In the current era, the occurrence of acute dissection of the ascending aorta in patients such as these will raise the question whether this was a failure of diagnosis, surveillance or decision making on the part of medical services.

In the context of aortic root surgery, if the threshold is set sufficiently low to minimise the risk of acute dissection, the population selected to have prophylactic surgery must inevitably include patients not destined to have AAD and who would have died with an intact ascending aorta. Recognition of this fact leads to a need to estimate an important but at present unavailable statistic: the number needed to treat $(\mathrm{NNtT})$. For patients with aortic root aneurysm at any given threshold for intervention, we should ideally know the NNtT to prevent a dissection and the $\mathrm{NNtT}$ to save a life. Consider as an example carotid endarterectomy which is performed to prevent stroke. For patients with $70 \%-99 \%$ carotid artery stenosis and who have had a transient ischaemic attack (the highest risk group for stroke), the $\mathrm{NNtT}$ is six, rising to 24 for $50 \%-69 \%$ stenosis. $^{i}$ These data are based on randomised trials. What is the $\mathrm{NNtT}$ at present day thresholds for root replacement? Because we intervene to prevent dissection, there are no contemporary data or prospective studies to guide us. As we have lowered the size threshold for intervention, the NNtT has necessarily increased.

\section{PROPHYLACTIC SURGERY ON THE AORTIC ROOT}

The most common form of aortic root aneurysm is that seen in Marfan syndrome. We recognise a characteristic morphology originating and

${ }^{\mathrm{i}} \mathrm{http}: /$ www.strokeupdate.org/ALLCURRENT/Consensus/ Consensus 2004/Cons carotis 2004.htm 


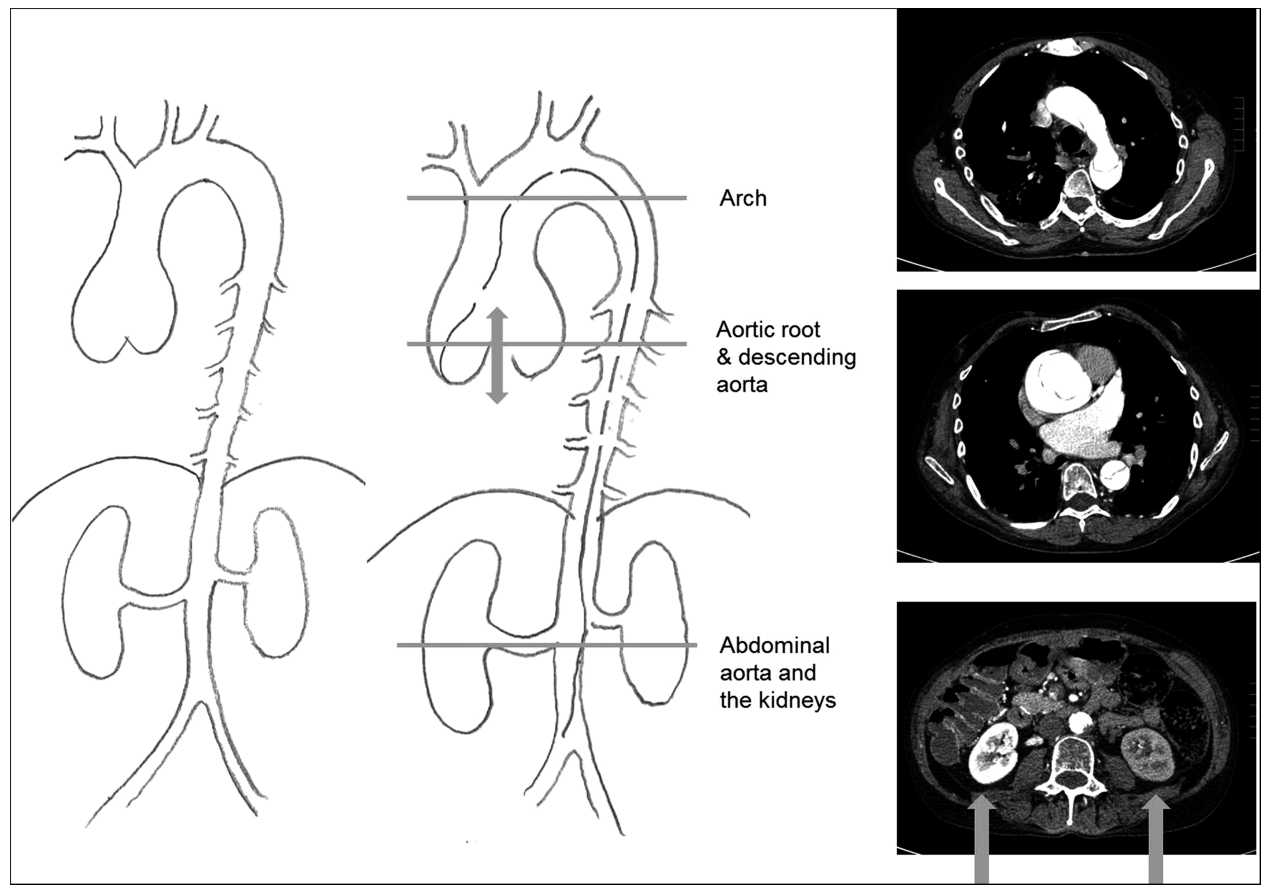

Figure 1 Acute aortic dissection in a man in his 50s from a family with Marfan syndrome and a history of several dissections and root replacements. One of the kidneys is not being perfused.

predominately affecting the sinuses of Valsalva, in which the expansion of the aorta includes the wall of the sinuses proximal to the coronary orifices (figure 3). In current practice, this form of aneurysm is amenable to confident echo diagnosis.

More complete morphological evaluation and measurement are readily achieved by either MRI or CT. In current practice, we recognise patients at risk of AAD by an enlarging aneurysm of the aortic root particularly with a family history of dissection. Replacement of the aortic root removes that specific risk. Distal segments of the aorta remain prone to aneurysmal dilatation, dissection and rupture.

The shape of the aortic root merits close attention (figure 4). The portion of the aortic wall proximal to the coronary orifices is characteristically elongated. This is not seen in ascending

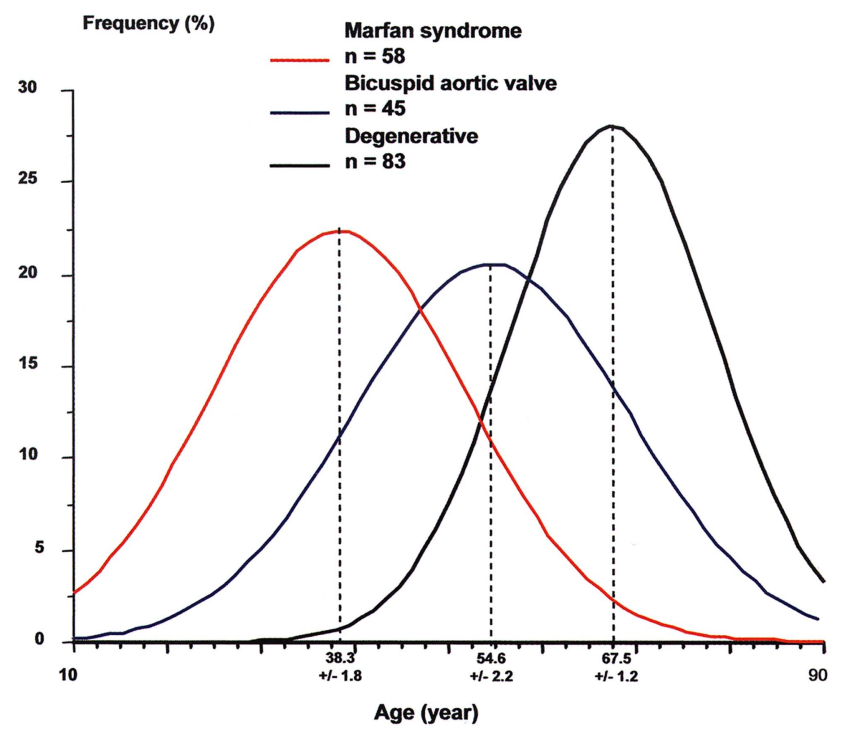

Figure 2 Relationship between age and the aetiology of aneurysms of the ascending aorta. ${ }^{1}$ aortic aneurysms associated with bicuspid aortic valve disease for example. The diameter at the level of closure of the aortic leaflets and its rate of change are the measurements used to make recommendations to the patient with respect to timing of aortic root surgery. Increasingly, the clinical diagnosis will be confirmed and augmented by knowledge of an individual's genome $e^{5}$ but this should not distract attention from the hazard represented by the aortic root disease. We argue here that patients with other aortopathies such as Ehlers-Danlos syndrome and Loeys-Dietz syndrome should generally be considered for aortic root surgery on similar imaging criteria, but in the latter perhaps at smaller size.

\section{Size threshold for prophylactic root replacement}

Current ESC/EACTS guidelines for the management of valvular heart disease state that, irrespective of the presence and severity of aortic valve regurgitation, surgery should be considered in patients with Marfan syndrome with risk factors (family history of dissection, size increase $2 \mathrm{~mm}$ /year in repeated examinations) who have aortic root disease with a maximum ascending aortic diameter of $\geq 45 \mathrm{~mm}$ (Class IIa, Level C evidence). ${ }^{6}$ The size criterion has come down from $60 \mathrm{~mm}$, through 55 and $50 \mathrm{~mm}^{7-10}$ to the present recommendation of $45 \mathrm{~mm} .{ }^{6}$ An increasing number of patients having root replacement were not destined to ever have have an $\mathrm{AAD}$, and hence our emphasis on needing to know the NNtT.

\section{THE CHOICE OF OPERATIONS}

Total root replacement

The current form of total root replacement (TRR) is with a conduit of low porosity woven Dacron incorporating a mechanical valve, usually bi-leaflet, with the coronary arteries anastomosed to the tube graft. There is the option of incorporating a tissue valve. ${ }^{11}$ This surgery has come a long way since the first operation by Bentall and De Bono in $1968 .{ }^{12}$ It is now a low risk, reproducible operation. 
Figure 3 Cardiac magnetic resonance images. Dark blood spin-echo image (left) and a diastolic frame from complete cine acquisition (right) of severe aortic root dilatation of typical Marfan morphology with type $A$ dissection and functional severe eccentric AR and LV dilatation. The aortic root diameter is $8 \mathrm{~cm}$.

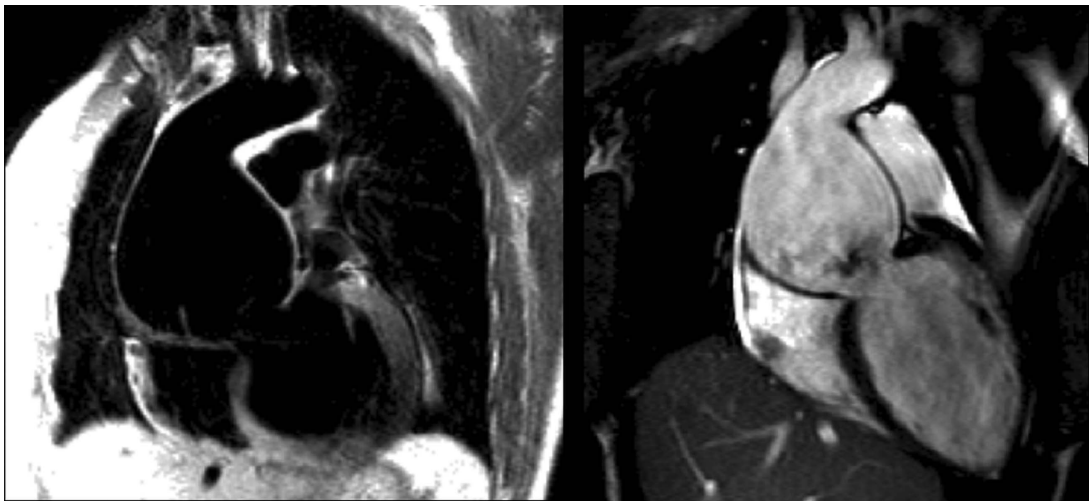

\section{Valve sparing root replacement}

It was the desire to spare patients the consequences of mechanical valve replacement that led Yacoub et $a l^{1314}$ and David et al ${ }^{15-17}$ to separately devise valve sparing root replacement (VSRR) operations, respectively, remodelling and reimplantation procedures. These are technically more demanding than TRR and revert to the 'workmanship of risk' where the surgeon has to make a series of judgements and unique adjustments typifying the craftsmanship of 'free hand' surgery. After 10 years of follow-up, the remodelling approach failed more often because the aortic annulus is not stabilised. In contrast, the results of the David procedure more often remain satisfactory to 15 years. In the last 10 years, there has been a growth of a variety of leaflet techniques and a classification system for aortic regurgitation based on the principles of Carpentier's classification of mitral regurgitation, which provides a universal vocabulary for communication. The dissemination of aortic valve repair has been slower than that for the mitral valve for two reasons. First, the surgeon's view of the aortic valve is from the outflow side making it impossible to visually assess and test the valve in its pressurised state and second the gradual recognition of the importance of two distinct structures in the aortic root, the ventriculo-aortic junction and the sino-tubular junction.

\section{Personalised external aortic root support}

In 2004, the first of a new form of operation was reported intended to prevent further growth in root aneurysms. ${ }^{18}$ This is quite different in concept from the two established procedures and is intended to be a prophylactic procedure. It has been evaluated in patients on average at an earlier stage in the natural history of MFS, in adults with aortic roots measuring between 40 and $50 \mathrm{~mm}$, without aortic regurgitation. The patient's own images are used to create a faithful copy of the aorta by computer aided design which is then made into a physical copy by '3D printing'. ${ }^{19}$ A macroporous fabric sleeve is then made which is placed around the aorta, including the segment proximal to the coronary arteries, down the aorto-ventricular junction (figure 5).

This swings the pendulum towards the 'workmanship of certainty': the surgeon is provided with a device which is made to measure for that individual's aorta and which will hold the aortic root at its existing shape and size. The process allows for a measured reduction in its diameter ${ }^{20}$ (figure 6). A formal

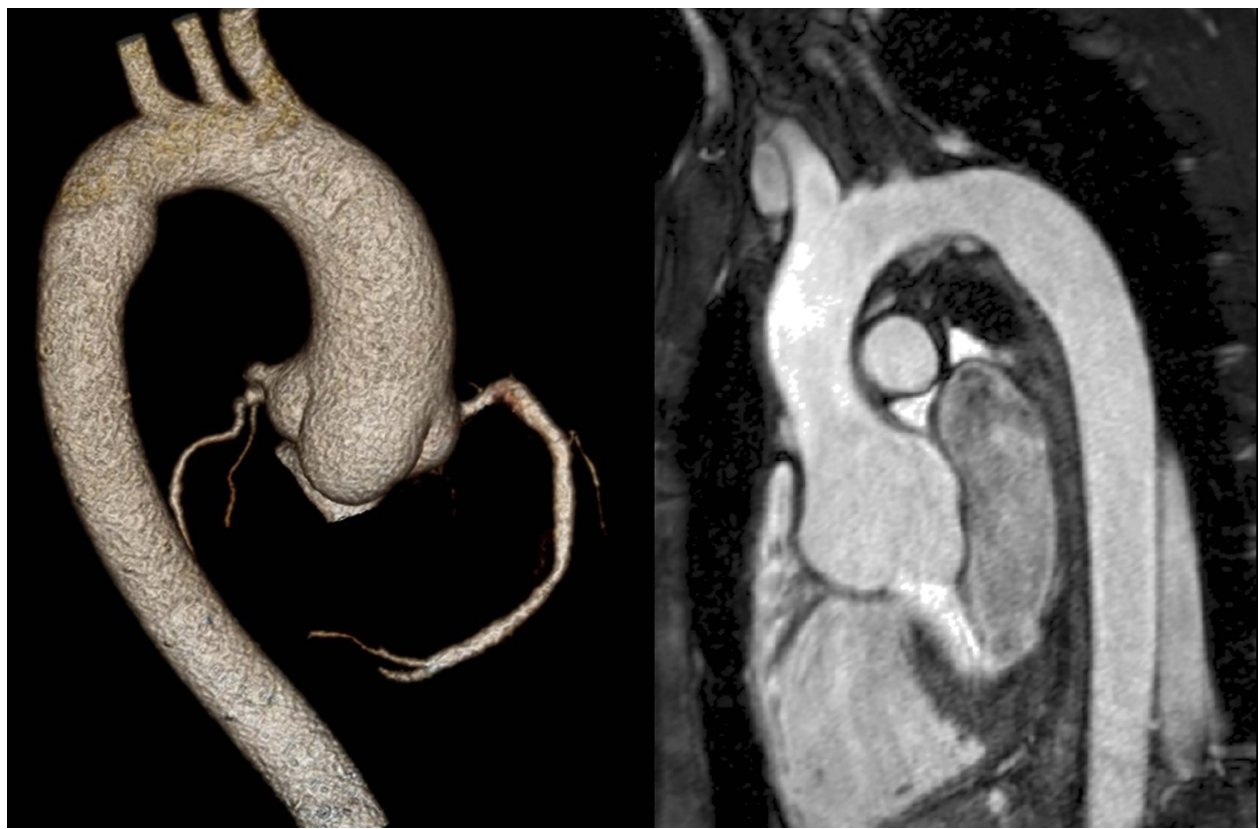

Figure 4 Aortic roots with the characteristic morphology and distal displacement of the coronary orifices but without gross enlargement. The CT image (left) is a volume rendered image of an ECG-gated scan of the thoracic aorta. The CMR image (right) is a thin section from a 3D steady-state in free precession sequence acquired in the left anterior oblique projection. 


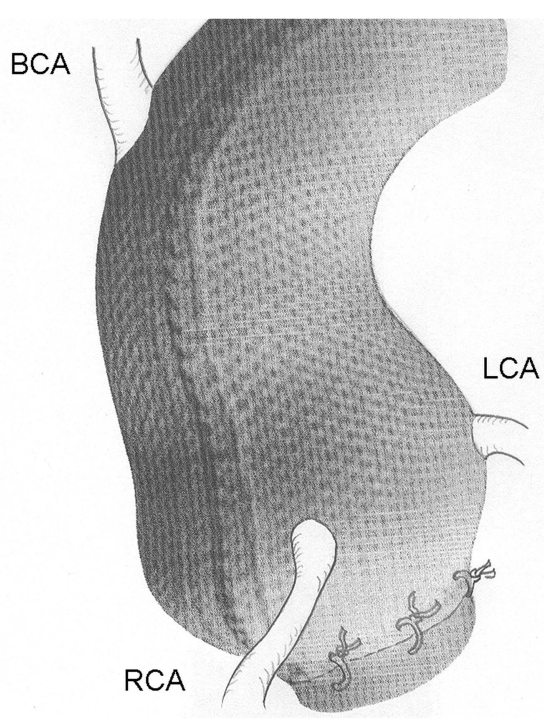

Figure 5 Personalised external aortic root support. BCA, brachiocephalic artery; LCA, left coronary artery; RCA, right coronary artery.

evaluation of whether aortic regurgitation can be reduced or eliminated is yet to be done but has been noted anecdotally.

The fabric, unlike the vascular grafts used in root replacement, is incorporated histologically creating a composite aortic wall which does not dilate further. ${ }^{21} 22$

Of its nature, this is not an option for patients who present with aortic dissection. It is unlikely to have a place in reoperation after aortic root surgery but could be an adjunct to protect other segments of the aorta. Personalised external aortic root support (PEARS) has been used as part of complex surgery in adult congenital heart disease and in combination with coronary artery surgery.

\section{COMPARATIVE ANALYSIS OF THE THREE PROPHYLACTIC OPERATIONS}

Procedural burden of care

The Aortic Valve Operative Outcomes in Marfan Patients (AVOOMP) study group provides prospectively collected data

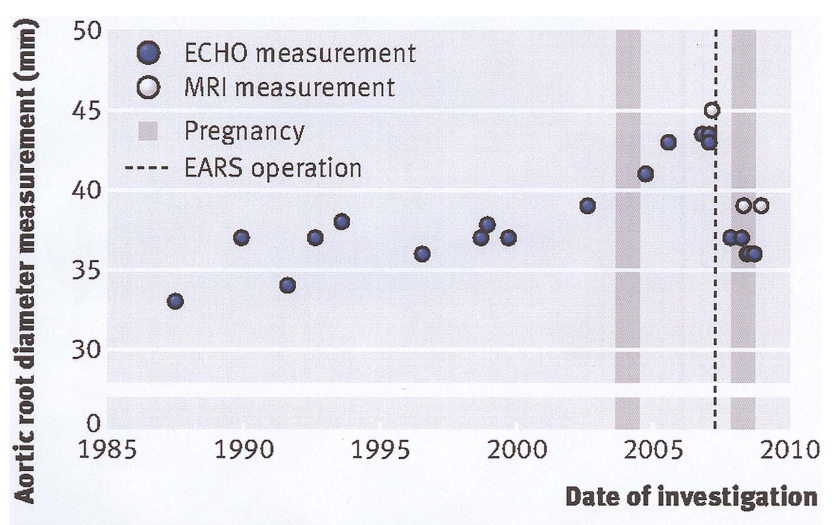

Allen, C. et al. BMJ 2010;340:c1692

Figure 6 Aortic root diameter over a 20-year period in a woman with Marfan syndrome; the diameter continued to enlarge during a first pregnancy and thereafter to reach the accepted threshold of $45 \mathrm{~mm} .^{28}$ She requested referral for personalised external aortic root support (PEARS). Her aortic dimensions were reduced as was seen in eight of the first 10 PEARS operations. ${ }^{20}$ on 239 patients having root replacement between 2005 and $2010 .^{23}$ For TRR $(\mathrm{N}=77)$ and VSRR $(\mathrm{N}=239)$, the median cardiopulmonary bypass times were 152 and $194 \mathrm{~min}$ and cross clamp times were 115 and $156 \mathrm{~min}$. Circulatory arrest was used in $29 \%$ and $20 \%$, respectively. It should be noted that the relatively few TRR operations were in older and higher risk patients reflecting preference for valve sparing surgery among these surgeons. In units where valve replacement is the usual and preferred operation, operative times for TRR are likely to be shorter. PEARS is routinely done without cardiopulmonary bypass, myocardial ischaemia or circulatory arrest. These procedural differences have been quantified in a comparative study. $^{24}$

\section{Risk of perioperative death}

In contemporary practice, in experienced units, the mortality for elective TRR in these patients, whose average age is midthirties, is under $1 \%$ and some teams now report that they have had no deaths for some years. In the AVOOMP study, 30-day mortality was $1 / 77$ for TRR $(1.3 \%$; $95 \%$ CIs $0.06 \%$ to $13 \%)$ and $1 / 239$ for VSRR $(0.04 \% ; 0.01 \%$ to $2 \%) .{ }^{23}$ The 34 th patient of 41 intended to have PEARS for Marfan syndrome died 5 days after surgery following an operative injury to the left main coronary artery $(1 / 41,2.4 \% ; 0.1 \%$ to $13 \%)$. He had severe pectus excavatum.

\section{Intention to treat and actual treatment}

In the AVOOMP study, ${ }^{23}$ in six patients $(6 / 239 ; 2.5 \%)$ there was a problem with sparing the aortic valve resulting in conversion to TRR with a composite valved root replacement. This outcome is usually not reported in clinical series of VSRR which are based on completed treatment rather than intention to treat analysis. Conversion from intended VSRR to TRR is generally more frequent than the AVOOMP rate of 1:40, particularly in small series and less experienced hands.

Among the patients in the AVOOMP study, cumulative, allcause aortic valve dysfunction at 1 year root replacement was recorded to be $23 / 219$ (10.5\%), of which were five intraoperative cases that were converted to mechanical AVR, one case of coronary artery kinking that required immediate reoperation (<30 days), one case of coronary pseudo aneurysm repair 6 days postoperative and 16 cases of AR $2+$ or greater (measured at 1 year postoperative). The total rate of major adverse valve-related events (MAVRE) at 1 year was 35/222 (15\%).

In the first year after operation, there was one further death in the TRR group (1/77, endocarditis) and two valve-related deaths in the VSRR patients (2/239). There was no significant difference in mortality or major adverse event rates for TRR or VSRR but the groups are not directly comparable and the nature of complications differs.

There has been one late death with PEARS 4.5 years after operation. The ascending aorta and the aortic valve were intact.

\section{Subsequent valve-related events}

With mechanical valve TRR, lifelong anticoagulation is mandatory and the long-term course with respect to the root replacement surgery is dominated by the continuing hazard of thrombotic events and anticoagulant-related bleeding. In a meta-analysis of 972 patients, the combined event rate for these complications was $0.7 \% .^{25}$ In the first year in the AVOOMP study where data were collected prospectively by study protocol, the thromboembolic and bleeding rates were recorded as $2 / 75$ $(2.7 \%)$ and $5 / 75(6.6 \%)$, respectively. ${ }^{23}$ 
Because the patients are in their mid-thirties on average, and many of them are in their teens and twenties, the impact of lifelong anticoagulation on quality of life is for some patients, considerable. It influences decisions about pregnancy, travel, work and recreation. Avoidance of these problems has been the driver towards VSRR.

The major concern with VSRR is the possibility of subsequent failure of the aortic valve. In both the meta-analysis by Benedetto and colleagues ${ }^{25}$ and the single institution report from Hannover, ${ }^{26}$ the re-intervention rate was $1.3 \%$ per annum. This means that for a 20 year old expecting to live to 70 or beyond, the likelihood of further surgery may be $65 \%$. Even this statistic understates the consequences of life affecting aortic regurgitation without re-intervention. Among the VSRR patients in the AVOOMP, the total rate of MAVRE was 65/225 (29\%). It is quite possible that mild degrees of aortic regurgitation can be overlooked especially if the patient has a sinus tachycardia, which will reduce the regurgitant fraction and will be well tolerated in young people.

At an average of over 4 years follow-up, there have been no instances of new or worsening regurgitation with PEARS.

\section{Effectiveness in preventing aneurysm and dissection}

Replacement of the ascending aorta precludes dissection in that segment of the aorta but not elsewhere. Although dissection has not been seen to date, PEARS cannot abolish the risk of dissection. The aorta is held at its preoperative or slightly smaller size so the major factor predisposing to dissection is obviated and this is likely to diminish the risk. The evidence of incorporation of the supporting mesh means that should dissection occur it would not lead to intrapericardial rupture which is the proximate cause of death in acute aortic dissection and the incorporation of the mesh will provide a technically more secure aortic wall at any subsequent operation than native Marfan aortic tissue.

The vulnerable sites for false aneurysm formation after TRR and VSRR are at suture lines, proximal and distal anastomosis of the tube graft, and around the coronary arteries. None of these sutured anastomoses are made in PEARS.

\section{Availability of the three procedures}

At present, PEARS is only available in a limited number of centres; four units have performed this surgery to date. TRR remains the commonest of the operations performed and is the most generally applicable form of surgery because it can be carried out irrespective of the size of the aorta or the degree of aortic valve regurgitation. For elective operations in young patients, the avoidance of lifelong anticoagulation and mechanical aortic valve-related thromboembolic events and endocarditis should be considered in referring patients for root replacement. They should be advised of the relative risks and benefits of valve sparing and valve replacing surgical alternatives.

\section{SELECTION CRITERIA FOR TRR, VSRR AND PEARS}

The recommended size criteria for intervention on the aortic root to avert dissection are based on the risk of further waiting balanced against the procedural risk of the surgery. The guidance from the European Society of Cardiology and the European Association for Cardio-Thoracic Surgery ${ }^{6}$ cites the review in the Lancet from $2005^{2}$ which in turn cites data from 1972. Better data are needed if we are to know the NNtT and if we are to have comparative effectiveness data and costeffectiveness for these three surgical approaches.
The question has been revisited in a decision analysis but unfortunately it gets us no further. ${ }^{27}$ The modellers had a wealth of data to use for survival after root replacement but for the critical estimate of survival without surgery they relied on five experts. The authors concluded that the gain in operating on an aorta of $5 \mathrm{~cm}$ diameter at the age of 20 is to increase life expectancy from 71 to 73 years. In the model, the estimated survival for patients with aortic root diameters of $6-7 \mathrm{~cm}$ was 68-69 years. This is very different to present beliefs about the risk of aortic root aneurysm so the model lacks face validity. It was of course an impossible question to answer. For example, the probability of being alive tomorrow is almost certainly better if you do not have your elective operation today so the exercise was heavily dependent on how the question was understood. These responses may have been informed by experience but did not provide the sort of information on which to build a robust mathematical model. What is more concerning is that there is no evidence to suggest that they were challenged for lack of face validity before publication.

\section{DIFFERING CRITERIA FOR TRR, VSRR AND PEARS}

TRR can be performed irrespective of aortic dimensions and the replacement valve is a secure and near certain means of correcting aortic valve regurgitation. A patient accepting lifelong anticoagulation, but not wanting ever to have further surgery, might choose this option.

In contrast, VSRR maintains normal physiology and freedom from anticoagulation and its attendant risks of bleeding. Failure to consistently achieve and maintain a competent aortic valve is the downside and there is a high lifetime probability of significant valve regurgitation leading to deteriorating function and/or further surgery.

PEARS seeks to include the benefits of VSRR but as a lesser procedure can be offered at smaller size and since it preserves the size and configuration of the aortic root optimises the chance of maintaining valve function. To date, there have been no instances of more than trivial aortic regurgitation. Results are too few and too early to know longer term outcomes.

As we seek to avoid any failure of our prophylactic policy, there will be a tendency to operate at lower size. One can argue for offering a valve sparing strategy at lower size in aortas such as those in figure 4. At present, we do not know how these decisions are made in clinical practice but it is likely that it is influenced by the beliefs of relative risk of the cardiologist and the technical preference of the surgeon, but it should also involve the patient who should be fully informed of the choices available.

The authors are actively seeking funding for a prospective evaluation of the surgical options available. This is provisionally called the Big Aortic Root Study. The tentative proposal is that this will incorporate an observational design similar to AVOOMP study ${ }^{23}$ with a randomised element in which patients eligible for either VSRR or PEARS are invited to have the decision made by random allocation.

Acknowledgements We are grateful to Raad Mohiaddan for cardiac magnetic resonance images and to Michael Rubens for CT Images. Steve Gallivan advised us on modelling with respect to Kim et al. ${ }^{27}$

Contributors TT and JP contributed equally to the first submission. JJMT reviewed and assisted in the revision and joined as an author.

Competing interests TT and JP due to their interest in Marfan syndrome and aortic surgery have been involved with the development and evaluation of personalised external aortic root support but have had no commercial involvement. JP has done this surgery only within the NHS facility where he works. 
Provenance and peer review Commissioned; externally peer reviewed.

Open Access This is an Open Access article distributed in accordance with the Creative Commons Attribution Non Commercial (CC BY-NC 3.0) license, which permits others to distribute, remix, adapt, build upon this work non-commercially, and license their derivative works on different terms, provided the original work is properly cited and the use is non-commercial. See: http://creativecommons.org/ licenses/by-nc/3.0/

\section{REFERENCES}

1 Michel J-B, Touat Z, Houard X, et al. New concepts in human abdominal aortic aneurysm pathophysiology: comparison with acending aortic and other locations. In: Sakalihasan N, Kuivaniemi H, Michel J-B, eds. Aortic aneurysms. Liege: Les Editions de I'Universite de Liege, 2008:165-85.

2 Murdoch JL, Walker BA, Halpern BL, et al. Life expectancy and causes of death in the Marfan syndrome. N Engl J Med 1972;286:804-8.

3 Silverman DI, Burton KJ, Gray J, et al. Life expectancy in the Marfan syndrome. Am J Cardiol 1995;75:157-60.

4 Judge DP, Dietz HC. Marfan's syndrome. Lancet 2005;366:1965-76.

5 Barrett PM, Topol EJ. The fibrillin-1 gene: unlocking new therapeutic pathways in cardiovascular disease. Heart 2013;99:83-90.

6 Vahanian A, Alfieri 0, Andreotti F, et al. Guidelines on the management of valvular heart disease (version 2012). Eur Heart J 2012;33:2451-96.

7 Treasure T. Elective replacement of the aortic root in Marfan's syndrome. Br Heart J 1993;69:101-3.

8 Treasure T, Reynolds C, Valencia O, et al. The timing of aortic root replacement in the Marfan syndrome: computer aided decision support. In: Enker J, ed. Cardiac surgery and concomitant disease. Darmstadt: Springer, 1999:91-8.

9 Treasure T, Chow T, Gallivan S. Replacement of the aortic root in Marfan's syndrome. N Engl J Med 1999;341:1473-4.

10 Treasure T. Cardiovascular surgery for Marfan syndrome. Heart 2000;84:674-8

11 Kirsch ME, Ooka T, Zannis K, et al. Bioprosthetic replacement of the ascending thoracic aorta: what are the options? Eur J Cardiothorac Surg 2009;35:77-82.

12 Bentall $\mathrm{H}$, De Bono A. A technique for complete replacement of the ascending aorta. Thorax 1968;23:338-9.

13 Fagan A, Pillai R, Radley-Smith $\mathrm{R}$, et al. Results of new valve conserving operation for treatment of aneurysms or acute dissection of aortic root. (Abstract). Br Heart $J$ 1983;49:302.

14 Yacoub MH, Gehle P, Chandrasekaran V, et al. Late results of a valve-preserving operation in patients with aneurysms of the ascending aorta and root. J Thorac Cardiovasc Surg 1998;115:1080-90.
15 David TE, Feindel CM. An aortic valve-sparing operation for patients with aortic incompetence and aneurysm of the ascending aorta. J Thorac Cardiovasc Surg 1992:103:617-21.

16 David TE, Armstrong S, Maganti M, et al. Long-term results of aortic valve-sparing operations in patients with Marfan syndrome. J Thorac Cardiovasc Surg 2009;138:859-64.

17 David TE. The aortic valve-sparing operation. J Thorac Cardiovasc Surg 2011;141:613-15.

18 Golesworthy T, Lamperth M, Mohiaddin R, et al. A jacket for the Marfan's aorta. Lancet 2004;364:1582.

19 Pepper J, Petrou M, Rega F, et al. Implantation of an individually computer-designed and manufactured external support for the Marfan aortic root. http://mmcts oxfordjournals org/cgi/content/full/mmt004?ijkey= WWjo5zbymFMuCWK\&keytype =ref 2013. http://mmcts.oxfordjournals.org/content/ 2013/mmt004.full.pdf+html

20 Pepper J, Golesworthy T, Utley M, et al. Manufacturing and placing a bespoke support for the Marfan aortic root: description of the method and technical results and status at one year for the first ten patients. Interact Cardiovasc Thorac Surg 2010;10:360-5.

21 Cohen O, Odim J, De la ZD, et al. Long-term experience of girdling the ascending aorta with Dacron mesh as definitive treatment for aneurysmal dilation. Ann Thorac Surg 2007;83:\$780-4.

22 Verbrugghe $\mathrm{P}$, Verbeken $\mathrm{E}$, Pepper J, et al. External aortic root support: a histological and mechanical study in sheep. Interact Cardiovasc Thorac Surg 2013;26:1-6

23 Coselli JS, Volguina IV, LeMaire SA, et al. Early and 1-year outcomes of aortic root surgery in patients with Marfan syndrome: a prospective, multicenter, comparative study. J Thorac Cardiovasc Surg 2014;147:1758-67.e4.

24 Treasure T, Crowe S, Chan KM, et al. A method for early evaluation of a recently introduced technology by deriving a comparative group from existing clinical data: a case study in external support of the Marfan aortic root. BMJ Open 2012;2: e000725.

25 Benedetto U, Melina G, Takkenberg JJ, et al. Surgical management of aortic root disease in Marfan syndrome: a systematic review and meta-analysis. Heart 2011;97:955-8.

26 Shrestha $\mathrm{M}$, Baraki $\mathrm{H}$, Maeding I, et al. Long-term results after aortic valve-sparing operation (David I). Eur J Cardiothorac Surg 2012;41:56-61.

27 Kim SY, Martin N, Hsia EC, et al. Management of aortic disease in Marfan Syndrome: a decision analysis. Arch Intern Med 2005;165:749-55.

28 Allen C, Pepper J. External aortic support for people with Marfan's syndrome. BMJ 2010;340:c1692. 